\title{
An Equisingular Deformation Theory via Embedded Resolution of Singularities
}

By

\author{
Yujiro KaWAMATA
}

In this paper we shall give a definition of equisingular deformations of isolated singularities and prove some results about it such as the existence of semi-universal deformations.

Roughly speaking, equisingularity means in this paper the existence of a simultaneous embedded resolution. Of course, in the case of plane curve singularities the definition coincides with Zariski's classical one.

In Section 1 we shall define equisingular deformation and prove some elementary properties of it. In Section 2 we shall study the deformation of locally product type of the pair obtained by the embedded resolution of singularity. In Section 3 we shall prove the existence theorem of semi-universal deformations.

The author would like to express his heartfelt thanks to Professor S. Iitaka, the conversations with whom were very fruitful and encouraging, and to Pf. M. Merle who pointed out to the author Example 2.

\section{\$1. Definitions}

Let $(X, o)$ be a germ of reduced complex space which is non-singular outside of $o$. By abuse of language, we sometimes write only $X$ instead of $(X, o)$ and mix up the concepts of a germ of complex space and a representative of it. Since $X$ is local, it admits a closed immersion $i: X \rightarrow A$ into a non-singular complex space $A$, where $\operatorname{dim} A=\operatorname{dim} m_{X, 0} / m_{X, 0}^{2}(A$ is also local). Denote $N$ $=\operatorname{dim} A$ and $n=\operatorname{dim} X$. Note that $N-n$ is not necessarily 1. Let us consider the following commutative diagram $(*)$ :

Communicated by H. Hironaka, March 16, 1978.

* Lehrstuhl für Mathematik, Universität Mannheim, 68 Mannheim A5, West Germany and Department of Mathematics, Faculty of Science, The University of Tokyo. 


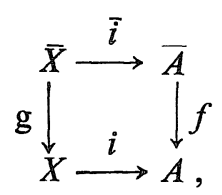

where $f$ is a point modification of $A$ at $o$ obtained by succession of monoidal transformations with non-singular centers, such that $\bar{X}=\operatorname{red} f^{-1}(X)$ is normal crossing in the sense of the following Definition 1. That is, $(*)$ is an embedded resolution of $X$ in $A$. Denote $g=f \mid \bar{X}$, and let $\bar{X}=Y \cup E$, where $Y$ is the strict transform of $X$ and $E=\operatorname{red} f^{-1}(o)$ is the exceptional locus. In particular,

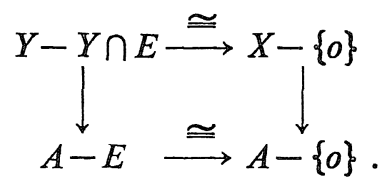

Definition 1. Let $A$ be a complex manifold and $X$ a closed analytic subset of $A . \quad X$ is called an analytic subset of normal crossing if the following conditions are satisfied:

(1) $X=\bigcup_{i=1}^{h} X_{i}$, where the $X_{i}(1 \leqq i \leqq h)$ are complex submanifolds of $A$.

(2) For each $p \in A$, there exists a neighborhood $U$ of $p$ and a system of local coordinates $\left\{z_{1}, \cdots, z_{n}\right\}$ on $U$ such that $X_{i}=\left\{z_{r_{i-1}+1}=\cdots=z_{r_{i}}=0\right\}$ for $1 \leqq i \leqq h$, where the $r_{i}$ are integers such that $-1 \leqq r_{i} \leqq n$ and $r_{i} \leqq r_{j}$ if $i \leqq j$, and we understand that $z_{0}=1$.

We note that for any $X$ there exists at least one such $(*)$ by [5].

Definition 2. An equisingular deformation (in this paper) of $X$ of type (*) consists of the following data:

(1) complex spaces $\bar{X}, \mathscr{X} S, \mathcal{A}$ and and a point $s_{0}$ of $S$,

(2) morphisms $p, q, I, I, F$ and $G$ which make up the following commutative diagram

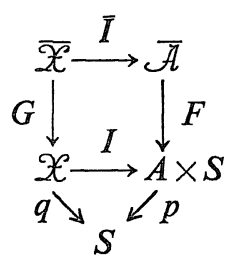

where $p$ is the projection, $q$ is flat, $I$, and $\bar{I}$ are closed immersions and $F$ is a 
modification of $A \times S$ along $\{0\} \times S$. We put $\bar{p}=p \circ F$ and $\bar{q}=q \circ G$.

(3) an isomorphism $\bar{\psi}$ from $(*)$ to the diagram obtained by the base change of $((*))$ by $\left\{s_{0}\right\} \rightarrow S$,

(4) we claim that $((*))$ is locally product in the sense of the following (such a deformation will be called also to be logarithmic cf. [7]): for every $x \in \mathcal{A}$ there is a neighborhood $U$ (resp. $V$ ) of $x$ in $\overline{\mathcal{A}}$ (resp. of $y=\bar{p}(x)$ in $S$ ) and an isomorphism $\alpha: U \rightarrow\left(U \cap \bar{p}^{-1}(y)\right) \times C$, which sends $U \cap \overline{\mathscr{X}}$ onto $\left(U \cap \overline{\mathscr{X}} \cap \bar{p}^{-1}(y)\right)$ $\times V$ and $\bar{p}=\operatorname{pr}_{V} \circ \alpha$.

Definition 3. A flat deformation $(q, \psi)$ of $X$, where $q: \mathcal{X} \rightarrow S$ is a morphism of complex spaces and $\psi: X \rightarrow \mathscr{X} \times_{S}\left\{s_{0}\right\}$ is an isomorphism, is said to be equisingular (in this paper) if there is some embedded resolution (*) of $X$ and an equisingular deformation of type $(*)$ which induces $(q, \psi)$.

Remark 1. (1) In case $n=1$ and $N=2$, our definition coincides with Zariski's original one.

(2) Our definition is strictly stronger than the one defined by J. Wahl [1 $\left.1_{5}\right]$ (see Example 1).

Remark 2. For any geometric fiber $X_{s}$ of $q, \operatorname{dim} m_{X_{s}, o_{s}} / m_{X_{s}, o s}^{2}=\operatorname{dim} A$. (This fact justifies the Definition 2.)

Proof. Since $q$ is flat, the dimension of the Zariski tangent space is upper semi-continuous. Suppose that this is not constant. By a base change we obtain the following situstion: (1) $S$ is a smooth curve, (2) There is a point $s_{0} \in S$ such that $\operatorname{dim} m_{X_{s}, 0_{s}} / m_{X_{s}, 0_{s}}^{2}$ is equal to $N_{1}$ if $s=s_{0}$ and to $N_{2}$ if otherwise, where $N_{1}>N_{2}$ are integers.

Let $L$ be the first infinitesimal neighborhood of the zero section $o \times S$ in $A \times S$, that is, the ideal of $L$ is the square of that of $o \times S$. Put $\mathscr{X}_{L}=\mathscr{X} \cap L$. By assumption, $\mathscr{X}_{L} \times{ }_{S}\left(S-\left\{s_{0}\right\}\right)$ defines an $\left(S-\left\{s_{0}\right\}\right)$-valued point $\alpha$ of the Grassmanian variety $G\left(N_{1}, N_{2}\right)$. Since the latter is proper and seperated, there is a unique $S$-valued point $\bar{\alpha}$ of $G\left(N_{1}, N_{2}\right)$ inducing $\alpha$. $\bar{\alpha}$ defines a subspace $V$ of $L$ inducing $\mathscr{X}_{L} \times{ }_{S}\left(S-\left\{s_{0}\right\}\right)$ on $L \underset{S}{ } \times\left(S-\left\{s_{0}\right\}\right)$. Then $\mathscr{X}_{L}=V$, which contradicts the assumption.

Q.E.D.

Proposition 1. Let $q: \mathscr{X} \rightarrow S$ be an equisingular deformation of $X$ where $S$ is reduced. Then there exists a homeomorphism $h: A \times S \rightarrow A \times S$ which sends $\mathscr{X}$ to $X \times S$ and $q=\operatorname{pr}_{S} \circ h$. 
Proof. Using a partition of unity, we obtain a diffeomorphism $\bar{h}: \bar{A} \rightarrow$ $\bar{A} \times S$ which sends each component of $\overline{\mathscr{X}}$ to the corresponding component of $\bar{X} \times S$ and $\bar{q}=\operatorname{pr}_{s} \circ \bar{h} . \quad \bar{h}$ is blown down to the desired $\mathrm{h}$.

Q.E.D.

Corollary 2. When $N=n+1$, i.e., in case of hypersurface singularities, any equisingular deformation preserves the Milnor number $\mu$.

Let $\mathscr{H}$ be a general family of hypersurfaces in $A$ parametrized by $S$. Let $H \subset A$ be the fiber of $\mathscr{H}$ over $s_{0}$. Let $\mathscr{X}_{\mathscr{H}}$ (resp. $X_{H}$ ) be the smallest closed analytic subset of $A \times S$ (resp. $A$ ) which induces $(\mathscr{X} \cap \mathcal{E})-(o \times S)$ (resp. $(X \cap H)-\{o\})$ on $(A-\{0\}) \times S$ (resp. $A-\{0\})$. They are called the strict general hyperplane section of $\mathscr{X}$ (resp. $X$ ) by $\mathscr{H}$ (resp. $H$ ).

Proposition 3. $\mathscr{X}_{\mathscr{G}} \rightarrow S$ is an equisingular deformation of $X_{H}$.

Proof. Trivial.

Corollary 4. When $N=n+1$, any equisingular deformation preserves $\mu^{*}$ of Teissier, hence satisfies the Whitney condition (cf. [11] and [12]).

Example 1. (Briançon-Speder [12]) $N=3$ and $n=2$.

$\mathrm{A}=$ Specan $\boldsymbol{C}\{x, y, z\}, S=$ Specan $\boldsymbol{C}\{t\}$ and $\mathscr{X} \subset A \times S$ is defined by the equation $x^{5}+y^{7} z+z^{9}+t x y^{6}=0$.

Then $q: \mathscr{X} \rightarrow S$ is (0) flat, (1) equisingular in the sense of $J$. Wahl [15], (2) $\mu$ is constant, but (3) $\mu^{*}$ is not constant, hence (4) not equisingular in the sense of this paper. Note that (5) $\mathfrak{X}_{\mathscr{M}}$ is not equisingular.

Remark 3. In case $N=n+1$, we do not know whether our definition is stronger or weaker than the $Z$-equisingularity in the sense of $J_{.}-P$. Speder [10].

For a fixed $X \subset \mathrm{A}$, there can be various embedded resolutions. Let

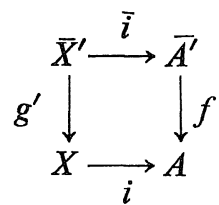

be another embedded resolution of $X \subset A$. Suppose that there is a morphism $\alpha: A \rightarrow A^{\prime}$ such that $f=f^{\prime} \circ \alpha$. Then $\alpha$ induces $\beta: \bar{X} \rightarrow \bar{X}^{\prime}$.

Proposition 5. Let $\mathscr{X}, S, \bar{X}, \overline{\mathcal{A}}, p$, q, etc. be an equisingular deformation of type (*) of $X \subset A$. Then there exists a neighborhood $S^{\prime}$ of $s_{0}$ in $S$ such that there exists an equisingular deformation $\mathfrak{X}^{\prime}, S^{\prime}, \overline{\mathcal{X}}^{\prime}, \overline{\mathcal{A}}^{\prime}, p^{\prime}, q^{\prime}$, etc. of type $(*)^{\prime}$ 
where $\mathfrak{X}^{\prime}=\mathfrak{X} \underset{S}{\times} S^{\prime}$ and $q^{\prime}=q \underset{S}{\times} S^{\prime}$.

Proof. We regard $A$ as an open set of the projective space $\mathbb{P}^{N}$. The point modifications $f$ and $F$ can be seen to be done for $\mathbb{P}^{N}$ and $\mathbb{P}^{N} \times S$, respectively. Since $f$ is blown down to $f^{\prime}, F$ can also blown down to some $F^{\prime}$ in some neighborhood $S^{\prime}$ of $s_{0}$ in $S$ by Theorem 3 of [7]. It is easy to check that $F^{\prime}$ induces an equisingular deformation of type $(*)$.'

Q.E.D.

Remark 4. There is no minimal embedded resolution even in case $N=3$

(a)

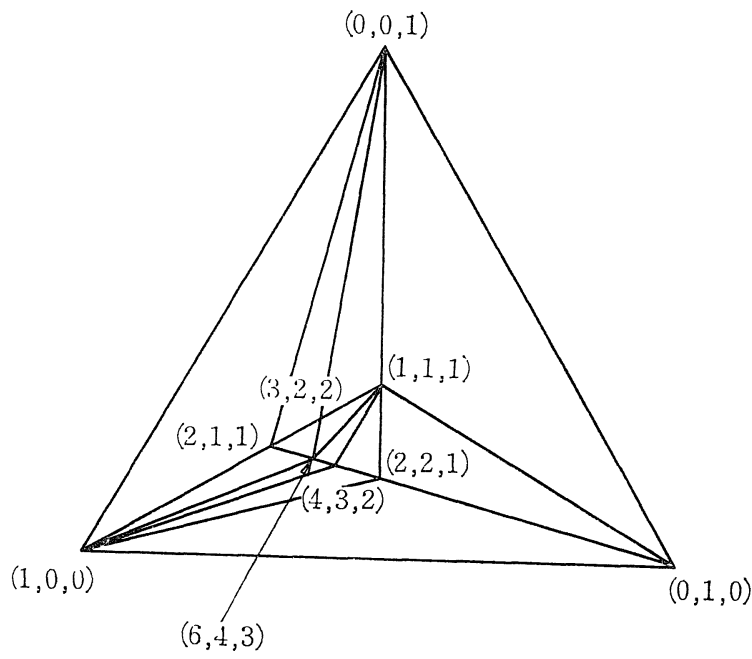

(b)

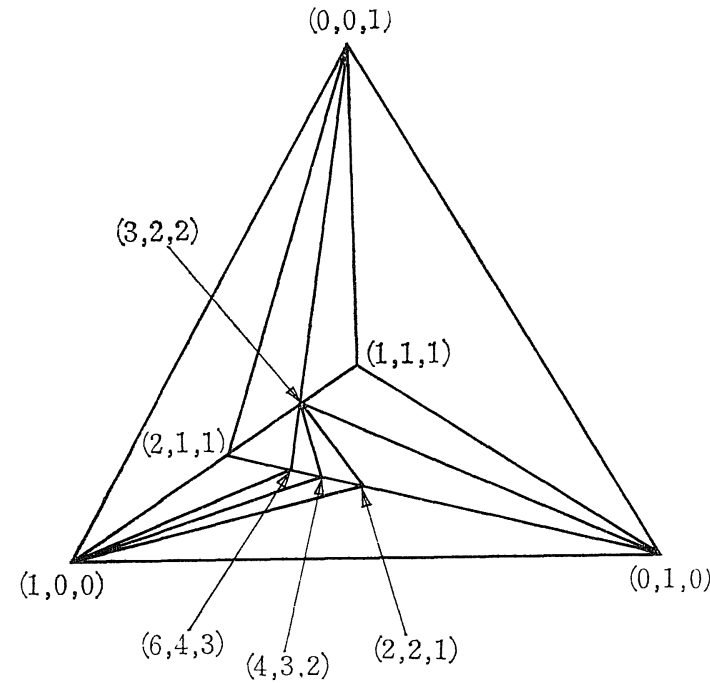

Figure 1 
and $n=2$. For example, let $X$ be defined by an equation $P=x_{1}^{2}+x_{2}^{3}+x_{3}^{4}$. Then there are two embedded resolutions corresponding to the two decompositions of positive cone of dimension 3 drawn in Figure 1. (See Example 2). We can easily chech that there is no embedded resolution which factors through both.

Example 2. (A. N. Varchenko [13]). Let $X$ be a hypersurface singularity defined by an equation $P=0$, and let $\Gamma(P)$ be the Newton polygon associated to $P$. $\quad P$ is said to be non-degenerate if for any facet $r \in \Gamma(P)$, the polynomials $x_{1} \frac{\partial P_{\gamma}}{\partial x_{1}}, \cdots, x_{N} \frac{\partial P_{\gamma}}{\partial x_{N}}$ have no common zero in $\left\{x \in \mathbb{C}^{N} ; x_{1}, \cdots, x_{N}=0\right\}$. In this case Varcenhko shows using the theory of toroidal embeddings that there is a modification $f: X(\Gamma) \rightarrow \mathbb{C}^{N}$ depending only on $\Gamma=\Gamma(P)$ such that $\operatorname{red} f^{-1}(X)$ is normal crossing. Hence we know that any small deformation of $P$ preserving the Newton polygon is equisingular with respect to $f$. However, for arbitrary complicated singularity which is usually degenerate, we should consider the geometric process of resolution, to which corresponds a sequence of Newton polygons.

\section{§2. Logarithmic Deformations}

Let us consider the situation $(*)$ of Section 1. For some time we shall set aside the lower half of $(*)$ and investigate the logarithmic deformation of $\bar{i}: \bar{X} \rightarrow A$, that is, the deformation which is locally product in the sense of Definition 2. We recall the definition of the logarithmic tangent sheaf (Definition 4 of [7]).

Definition 4. Let $\bar{A}$ be a complex manifold and $X$ a closed analytic subset of $A$. The logarithmic tangent sheaf $T_{A}(\log X)$ is the subsheaf of the tangent sheaf $T_{A}$ consisting of derivations which sends the ideal sheaf of $X$ in $A$ into itself.

By the general theory of deformations of manifolds (Exp. III of [4]), the infinitesimal deformation of $\bar{A} \subset \overline{\mathcal{A}}$ is described by the cohomology groups of $T_{\bar{A}}(\log \bar{X})$ as follows (cf. $\S 1$ of [7]): (1) $H^{1}\left(\bar{A}, T_{\bar{A}}(\log \bar{X})\right.$ ) is the set of isomorphism classes of all deformations over $S=$ Specan $\mathbb{C}[x] /\left(x^{2}\right)$, (2) The obstruction of extending the deformation lies in $H^{2}\left(\bar{A}, T_{\bar{A}}(\log \bar{X})\right.$ ) (Note that since $X$ is a germ, $H^{i}\left(\bar{A}, T_{\bar{A}}(\log \bar{X})\right)=R^{i} f_{*} T_{\bar{A}}(\log \bar{X})$ for $i>0$.)

Let 


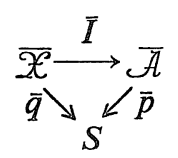

be a logarithmic deformation of $\bar{i}: \bar{X} \rightarrow A$. Replacing neighborhood of $s_{0}$ in $S$, if necessary, $\bar{A}$ can be blown down to $A \times S$. Denote by $F: \bar{A} \rightarrow A \times S$ the canonical morphism. $F$ induces an isomorphism $\overline{\mathscr{A}}-\mathcal{E} \rightarrow(A-\{o\}) \times S$. Let $\mathscr{X}=\mathscr{X}_{S}$ be the smallest subspace of $A \times S$ inducing $\bar{X} \cap(\overline{\mathscr{A}}-\mathcal{E})$ in $(A-\{o\}) \times S$. $\overline{\mathscr{X}}_{S}$ is called the strict image of $\overline{\mathscr{X}}$ over $S$.

Proposition 6. Under the situation above, there exists a germ $T$ of closed analytic subset of $S$ at $s_{0}$ satisfying the following conditions: If $Z$ is a closed subspace of $S$ such that the strict image $\mathfrak{X}_{Z}$ of $\bar{X} \times \underset{S}{Z}$ over $Z$ is a flat deformation of $X$ over $Z$, then the germ of $Z$ at $s_{0}$ is contained in $T$.

We need the following

Lemma. $\mathscr{X}_{Z}$ is a flat deformation of $X$ over $Z$ if and only if the geometric fiber $\mathscr{X}_{Z} \times{ }_{Z}\left\{s_{0}\right\}$ of $\mathscr{X}_{Z}$ over $s_{0}$ is reduced, hence isomorphic to $X$ (we shall say shortly that $Z$ satisfies the property $(H)$ in this case).

Proof. Put $C=\mathcal{O}_{Z, s_{0}}, D=\mathcal{O}_{A \times Z}, E=\mathcal{O}_{\mathfrak{X}_{Z}}=D / I$ and $m=m_{z, s_{0}}$. There is an exact sequence

$$
0 \rightarrow \operatorname{Tor}_{1}^{C}(C / m, E) \rightarrow m \otimes E \rightarrow E
$$

Let $a_{1}, \cdots, a_{h}$ be elements of $m$ which induces a basis of $m / m^{2}$. Suppose that an element $f=\sum_{i=1}^{h} a_{i} \otimes\left[f_{i}\right]\left(f_{i} \in D\right)$ of $m \otimes E$ is mapped to zero in $E$. Considering the values of the functions on $\bar{A}_{Z}$, we conclude that $f_{i}$ is in $(m D, I)$. Therefore, replacing the representatives $f_{i}$ of $\left[f_{i}\right]$ by suitable ones, we may suppose $f_{i} \in m D$, that is, $f_{i}=\sum_{j=}^{h} a_{j} g_{i j}$ for some $\mathrm{g}_{i j} \in D$. By a similar argument, we may sup pose $g_{i j}$ is in $m$, and so on. Hence $f$ is in the image of $\left(\cap m^{k}\right) \otimes E$, and the latter is zero. Thus, $\operatorname{Tor}_{1}^{C}(C / m, E)=0$, which shows the lemma.

Q.E.D.

Proof of the Proposition 6. We have to show the following two claims:

(1) If $Z_{1}$ and $Z_{2}$ satisfy $(H)$, then $Z_{1} \cup Z_{2}$ also satisfies $(H)$.

(2) If an ascending chain of subspaces $Z_{1} \subset Z_{2} \subset \cdots$ satisfy (H) and $Z$ is the smallest closed subset of $S$ containing all the $Z_{i}$, then $Z$ satisfies $(H)$. 
Proof of (1). As long as the germs are concerned, $Z$ satisfies $(H)$ if and only if every function $f_{0}$ in $\mathcal{O}_{\bar{A}}(-\bar{X})$ can be extended to some $f$ in $\mathcal{O}_{\overline{\mathcal{A}}_{Z}}\left(-\overline{\mathscr{X}}_{Z}\right)$, where $\overline{\mathscr{A}}_{Z}=\overline{\mathscr{A}} \times \underset{S}{ } Z$ and $\overline{\mathscr{X}}_{Z}=\overline{\mathscr{X}} \underset{S}{\times} Z$. Since $\mathscr{X}_{Z_{1}} \times{ }_{Z_{1}}\left\{s_{0}\right\}$ is reduced, $\mathscr{X}_{Z_{1}} \times$ $\left(Z_{1} \cap Z_{2}\right)$ has no function whose support is on the zero section. Therefore, every function $f_{12}$ in $\mathcal{O} \overline{\mathcal{A}}_{z_{1} \cap z_{2}}\left(-\overline{\mathscr{X}}_{Z_{1} \cap z_{2}}\right)$ can be extended to some $f_{1}$ in $\mathcal{O} \overline{\mathcal{A}}_{z_{1}}$ $\left(-\overline{\mathscr{X}}_{Z_{1}}\right)$. Now, starting from $f_{0}$ we find $f_{12}$ over $Z_{1} \cap Z_{2}$, and then $f_{1}$ and $f_{2}$ over $Z_{1}$ and $Z_{2}$, respectively. They patch together to the desired $f$.

Proof of (2). Let $I_{j}$ be the ideal of $Z_{j}$ in $Z$ for $j=1,2, \cdots$ and $m=m_{z, s_{0}}$. Since for any $k \in Z$ there is some $j$ such that $m^{k} \supset I_{j}$, (2) is equivalent to the following

(2 bis) If $\hat{Z}$ satisfies $(H)$, then $Z$ satisfies $(H)$, where $\hat{Z}$ is the completion of $Z$ by the ideal $m$.

Proof of ( 2 bis). We have only to prove that the completion $\hat{\mathfrak{X}}_{Z}=$ proj-lim $\mathscr{X}_{Z} \times Z / m^{k}$ of $\mathscr{X}_{Z}$ with respect to the ideal $m \Theta_{\mathfrak{X}_{Z}}$ has no function whose support lies on the zero section. For, if this is shown, then $\hat{\mathscr{X}}_{Z}$ is flat over $Z$ by the assumption and hence $\hat{\mathscr{X}}_{Z} \times\left\{s_{\hat{Z}}\right\}=\mathscr{X}_{Z} \times\left\{s_{\hat{Z}}\right\}$ is reduced.

By assumption, for any general hyperplane defined by the equation $R=0$,

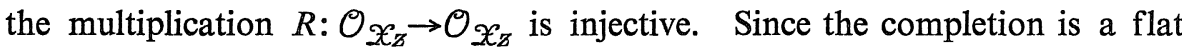
operation, $R: \mathcal{O}_{\hat{\mathfrak{X}}_{Z} \rightarrow \mathcal{O}_{\hat{X}_{Z}}}$ is also injective, which shows (2 bis). Thus the proof of Proposition 8 is completed.

Q.E.D.

Proposition 7. If $X$ is normal, then the $T$ in Proposition 6 is equal to $S$.

Proof. By proposition 6, we may assume that $S=$ Specan $C[t] /\left(t^{h}\right)$ for some integer $h$. Let $f_{0}$ be any element of $\mathcal{O}_{\bar{A}}(-\bar{X})$. We shall denote by the same letter the trivial extension along $S$. Since $f_{0}=0$ on $\bar{X}$, there is some $f_{1}$ in $\mathcal{O}_{\bar{X}}$ such that $f_{0}=t f_{1}$ on $\overline{\mathscr{X}}$. Since $X$ is normal, the restriction of $f_{1}$ to $\bar{X}$ is also a function on $X$. Let $g_{1}$ be a representative of it in $\mathcal{O}_{A}$. Since $g_{1}=f_{1}$ on $\bar{X}$, there is some $f_{2}$ in $\mathcal{O}_{\bar{X}}$ such that $f_{1}=g_{1}+t f_{2}$, hence $f_{0}-t g_{1}=t^{2} f_{2}$. Repeating the same process as above, we shall at last obtain elements $g_{1}, \cdots, g_{h-1}$ of $\Theta_{A}$ (or $\mathcal{O}_{A \times S}$ ) such that $f_{0}-t g_{1}-t^{2} g_{2}-\cdots-t^{h-1} g_{h-1}=0$ on $\overline{\mathscr{X}}$. The left hand side of the equality gives the desired extension of $f_{0}$.

Q.E.D.

Corollary 8. When $N=n+1, T=S$.

Proof. When $n$ is greater than one, $\mathrm{X}$ is normal. When $n=1$, the Corollary will be proved in Section 3 (see proof of Corollary 10.) 
Example 3. (F. Pham p.190 of [3]). $N=2$ and $n=1 . \quad A=\operatorname{Specan} C\left\{x_{1}, x_{2}\right\}$ and $X=$ Specan $\boldsymbol{C}\left\{x_{1}, x_{2}\right\} /\left(x_{1}^{3}+x_{2}^{9}\right)$. By a minimal embedded resolution of $X$, we obtain $\bar{A}, \bar{X}$, etc. Then, $\operatorname{dim} H^{1}\left(A, T_{\bar{A}}(\log \bar{X})\right)=2$. A semi-universal deformation is given by $S=$ Specan $\mathbb{C}\left\{t_{1}, t_{2}\right\}$ and $\mathfrak{X}=$ Specan $\mathbb{C}\left\{x_{1}, x_{2}, t_{1}, t_{2}\right\} /$ $\left(x_{1}^{3}+x_{2}^{9}+t_{1} x_{1} x_{2}^{6}+t_{2} x_{1} x_{2}^{7}\right)$ in $A \times S$. For each $t=\left(t_{1}, t_{2}\right)$ in $S, \operatorname{dim} H^{1}\left(\bar{A}_{t}, T_{\bar{A}_{t}}\right.$ $\left(\log \bar{X}_{t}\right)$ ) equals to 2 if $t_{2}=0$, and to 1 if $t_{2} \neq 0$. On the other hand, $F$. Pahm has shown that the singularity along the line $t_{1}=0$ is different from that along $t_{1} \neq 0$ in connection with the versal flat deformations.

\section{§3. Semi-Universal Deformations.}

In this section we shall prove the following

Theorem 1. Consider the situation (*). Then there exists a semi-universal object for all equisingular deformations of type (*), that is, there exists an equisingular deformation $\mathfrak{X}, S, \bar{X}, \bar{A}, p$, q, etc. of type (*) such that for any equisingular deformation $\mathfrak{X}^{\prime}, S^{\prime}, \overline{\mathfrak{X}}^{\prime}, \overline{\mathcal{A}}^{\prime}, p^{\prime}, q^{\prime}$, etc. of type (*), when we replace $S^{\prime}$ by a suitable neighborhood of $s_{0}^{\prime}$ if necessary, there is a morphism $\alpha: S^{\prime} \rightarrow S$ and $\mathfrak{X}^{\prime}$, $\bar{X}^{\prime}, p^{\prime}, q^{\prime}$, etc. are obtained by the pull-back of $\mathscr{X}, \bar{X}, p$, $q$, etc. by $\alpha$, and moreover the tangent map $T_{\infty}: T_{S^{\prime}, s_{0}^{\prime}} \rightarrow T_{S, s_{0}}$ is uniquely determined.

Proof. By Proposition 6, we have only to prove the existence of a semiuniversal logarithmic deformation of $\bar{i}: \bar{X} \rightarrow \bar{A}$.

As in the proof of Proposition 5, embed $\bar{A}$ in a point modification $Z$ of a projective space $\boldsymbol{P}^{N}$. By Theorem 1 of [7], there exists a semi-universal logarithmic deformation

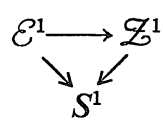

of $\underset{E \rightarrow Z}{\stackrel{i}{\rightarrow}}$. Over this we shall construct the desired deformation.

First, we shall prove the theorem under the condition that $n$ is greater than one. Let $E=\bigcup_{i=1}^{M} E_{i}$ be the irreducible decomposition and $a=\left(a_{1}, \cdots, a_{M}\right)$ a sequence of non-negative integers. Denote by $E_{a}$ (resp. $Y_{a}, \mathcal{E}_{a}^{1}, q_{a}^{1}$ ) the infinitesimal neighborhood of $E$ (resp. $Y \cap E, \varepsilon^{1}, q y^{1} \cap \varepsilon^{1}$ ) of order $a_{i}$ along $E_{i}$ (resp. $E_{i}, \mathcal{E}_{i}^{1}, \mathcal{E}_{i}^{1}$ ). Applying the relative Douady theorem (Theorem 2 of [9]) for $S^{1}, \mathcal{E}_{a}^{1}$ and $\mathcal{O}_{\mathcal{E}_{a}^{1}}$ instead of $S, X$ and $\mathcal{E}$ there, respectively, we obtain a universal family $\mathcal{U}_{a}^{2} \rightarrow \mathcal{E}_{a}^{1} \times H_{S^{1}} H_{S^{1}}\left(\Theta_{\mathcal{E}_{a}^{1}}\right)$ over a complex space $H_{s^{1}}\left(\Theta_{\mathcal{E}_{a}^{1}}\right)$. We consider 
a small enough neighborhood in $H_{S^{1}}\left(\mathcal{O}_{\mathcal{E}_{a}^{1}}\right)$ of the point corresponding to $Y_{a} \cap E_{a}$. Then there is a closed analytic subset $V_{a}$ of $H_{s^{1}}\left(\mathcal{O}_{\mathcal{E}_{a}^{1}}\right)$ near that ponit which represents the flat families of locally product type in the sense of Definition 2. On the other hand, the tangent space of the functor of the deformation of locally product type is $H_{a}^{1}=H^{1}\left(A, T_{\bar{A}}\left(\log E_{a}\right)\left(\log Y_{a}\right)\right)$ by Exp. III of [4], where $T_{\bar{A}}\left(\log E_{a}\right)\left(\log Y_{a}\right)$ is the intersection of $T_{\bar{A}}\left(\log E_{a}\right)$ and $T_{\bar{A}}\left(\log Y_{a}\right)$ in $T_{\bar{A}}$. Therfore, there is a canonical surjection $T_{V_{a}} \rightarrow H_{a}^{1}$. Let $M_{a}$ be the kernel and $L_{a}$ some complementary space of it in $T_{V_{a}}$. We may suppose that $V_{a}$ is embedded in a linear space $W_{a}=T_{V_{a}}$ and $L_{a}$ is a linear subspace of $W_{a}$. Put $S_{a}=V_{a} \cap L_{a}$ and let $\mathscr{J}_{a} \subset \mathcal{E}_{a} \subset \overline{\mathcal{A}}_{a}$ be the induced family over $S_{a}$, where $\mathcal{E}_{a}$ $=\mathcal{E}_{a}^{1} \times S_{S^{1}}$ and $\bar{\AA}_{a}=\overline{\mathcal{A}}^{1} \times S_{S^{1}} S_{a}$.

Lemma. $\quad \mathscr{y}_{a} \subset \mathcal{E}_{a} \subset \bar{A}_{a}$ over $S_{a}$ gives a semi-universal deformation of locally product type of $Y_{a} \subset E_{a} \subset \bar{\AA}$.

Proof. Let $G$ be the automorphism group of an infinitesimal nieghborhood of some order $k$ of the origin of $A . \quad G$ acts on $V_{a}$ if $k$ is large enough. Let $a y^{\prime} \subset \mathcal{E}^{\prime} \subset \bar{A}^{\prime}$ over $B$ be any deformation of locally product type of $Y_{a} \subset E_{a} \subset A$. By the universality of the relative Douady space, there is a canonical morphism $\sigma: B \times G \rightarrow V_{a} \subseteq W_{a}$. If the order $k$ is sufficiently large, $o \times T_{G}$ is sent onto $M_{a}$ by $T_{\sigma}$. Applying the implicit function theorem to the composite morphism $B \times G \stackrel{\sigma}{\rightarrow} W_{a} \rightarrow W_{a} / L_{a}=M_{a}$, we obtain a morphism $s: B \rightarrow G$ such that $\sigma(b, s(b))$ falls into $L_{a}$ for all $b$ near the origin. This proves the Lemma. Q.E.D.

Note that $S_{a}$ is an $S^{1}$-complex space and if $a \leqq b$, then there is a canonical morphism $S_{a} \rightarrow S_{b}$. By the exact sequence of sheaves:

$$
0 \rightarrow T_{\bar{A}}(\log \bar{X}) \rightarrow T_{\bar{A}}\left(\log E_{a}\right)\left(\log Y_{a}\right) \rightarrow N_{Y}\left(-Y_{a}\right) \rightarrow 0,
$$

where $N_{Y}$ is the normal sheaf of $Y$ in the sense of Proposition 1 of [7], we obtain the following exact sequence:

$$
\begin{aligned}
& H^{0}\left(\bar{A}, T_{\bar{A}}\left(\log E_{a}\right)\left(\log Y_{a}\right)\right) \rightarrow H^{0}\left(Y, N_{Y}\left(-Y_{a}\right)\right) \rightarrow H^{1}\left(A, T_{\bar{A}}(\log \bar{X})\right) \\
& \rightarrow H^{1}\left(\bar{A}, T_{\bar{A}}\left(\log E_{a}\right)\left(\log Y_{a}\right)\right) \rightarrow H^{1}\left(Y, N_{Y}\left(-Y_{a}\right)\right) .
\end{aligned}
$$

We assume that $a=\left(a_{1}, \cdots, a_{M}\right)$ is large enough and the $a_{i}$ have some appropriate ratioes each other. Then $H^{1}\left(Y, N_{Y}\left(-Y_{a}\right)\right)$ vanishes by Serre's theorem. On the other hand, the image of the map $H^{0}\left(\bar{A}, T_{\bar{A}}\left(\log E_{a}\right)\left(\log Y_{a}\right)\right) \rightarrow$ $H^{0}\left(Y, N_{Y}\left(-Y_{a}\right)\right)$ coincides with the intersection of $H^{0}\left(Y, N_{Y}\left(-Y_{a}\right)\right)$ and the image of the map $H^{0}\left(\bar{A}, T_{\bar{A}}(\log E)\right) \rightarrow H^{0}\left(T, N_{Y}\right)$. Thus the former is surjective 
under the same kind of the conditions on the $a_{i}$ as above. Therefore, under the assumptions above, $H^{1}\left(\bar{A}, T_{\bar{A}}(\log \bar{X})\right)$ is mapped bijectively onto $H^{1}(A$, $\left.T_{\bar{A}}\left(\log E_{a}\right)\left(\log Y_{a}\right)\right)$. This implies that the canonical map $S_{a} \rightarrow S_{b}$ is a closed immersion (near the origin) for such $a$ and $b, a \geqq b$. Since the local ring of the complex space is noetherian, we finally conclude that (the germ of) the spaces $S_{a}$ are stationary for large $a$ and appropriate ratioes. Denote by $S$ the space thus obtained. Put $\mathcal{E}=\mathcal{E}^{1} \underset{S^{1}}{\mathcal{S}}$ and $\overline{\mathscr{A}}=\overline{\mathscr{A}}^{1} \times S$.

Summing up, we have obtained a complex space $S$ over which a sequence $\left\{y_{a} \subset \mathcal{E}_{a} \subset \overline{\bar{A}}\right\}$ of semi-universal deformations stands. We have to construct analytic spaces from the data given above. Let $\hat{A}_{S}$ be the completion of $A_{S}$ $=A \times S$ along $o \times S$. Then, $\hat{A}=\bar{A} \times \hat{A}_{S} \hat{A}_{S}$ is isomorphic to the completion of $\bar{A}$ along $\mathcal{E}$. We have a subspace $\mathcal{Y}$ of $\mathcal{A}$ corresponding to the sequence $\left\{\mathcal{I}_{a}\right\}$. Note that there is a canonical isomorphism from $\overline{\mathscr{A}}-\varepsilon$ to $\hat{A}_{S}-(o \times S)$. Let $\hat{X}$ be the smallest subspace of $\hat{A}_{S}$ which induces $\mathscr{Y}-\varepsilon \cap \mathscr{Y}_{S}$ in $\hat{A}-(o \times S)$. By Theorem 1.4 of [1] and using the technique of Chap er II of [2], for arbitrary number $c$ we obtain a complex subspace $\mathscr{X}$ of $A_{S}$ which induces the same thing as $\mathscr{X}$ on the $c$-th infinitesimal neighborhood of $o \times S$ in $A_{S}$. Then by the main theorem of [6], if $c$ is large enough, the completion of $\mathscr{X}$ along $o \times S$ is isomorphic to $\hat{\mathscr{X}}$. We shall show that the space $\mathscr{X}$ thus obained is the desired semiuniversal deformation.

Let $\mathscr{X}^{\prime} \rightarrow B$ (and $\overline{\mathscr{X}}^{\prime}$, etc.) be any other equisingular deformation of type (*). For large $c$, the $c$-th infinitesimal neighborhood $\mathscr{X}_{0}^{\prime}$ is isomorphic to the pull back of $\mathscr{X}_{c}$ by a morphism $\alpha_{c}: B \rightarrow S$. By [6] again, $\mathscr{X}^{\prime}$ is isomorphic to the pull back of $\mathscr{X}$ by $\alpha=\alpha_{c}$. Thus, the proof of the Theorem 1 in case of $n \geqq 2$ is completed.

In case $n=1$, there is nothing difficult. The movements of the curve segments $\mathscr{X}^{1}$ in $\bar{\AA}^{1}$ have no obstructions, since they are strictly local. We can make all at once the semiuniversal family instead of the step-by-step construction above.

Q.E.D.

Corollary 9. If $S=T$ in the notation of Proposition 6 and $H^{2}\left(\bar{A}, T_{\bar{A}}(\log \bar{X})\right)$ $=0$, then there exists a semi-universal equisingular deformation of type (*) whose base space is non-singular.

Corollary 10. If $N=2$ and $n=1$, then there exists a semi-universal equisingular deformation whose base space is non-singular.

Proof. Let $(*)^{m}$ be the minimal embedded resolution of $X$. Since any 
equisingular deformation is obtained as of type $(*)^{m}$ by Proposition 5 , we may restrict our attension to the situation $(*)^{m}$. Since the point blowing ups have no obstructions, the base space $S^{m}$ of the semi-universal logarithmic deformation of $\bar{X}^{n} \subset \bar{A}^{m}$ is non-singular. Then the space $\mathscr{X}^{m}=\mathscr{X}_{S^{m}}^{m}$ obtained by a bolwing down is a Cartier divisor in $A \times S^{m}$. Therefore, the geometric fiber $\mathscr{X}^{m} \times\left\{s_{0}\right\}$ is also a Cartier divisor and hence reduced. Thus $\mathscr{X}^{m}$ is flat over $S^{m}$. On the other hand, the cinsideration of dimensions shows that $H^{2}(\bar{A}$, $\left.T_{\bar{A}}(\log \bar{X})\right)=0$. Thus Corollary 10 is proved by Corollary $9 . \quad$ Q.E.D.

Proof of Corollary 8 continued. Since any $\mathfrak{X}_{Z}$ (in the notation of Proposition 6) is obtained from $\mathscr{X}^{m}$ by a pulling back, $\mathscr{X}_{Z}$ is flat.

Q.E.D.

Remark 5. Corollary 10 was first obtained by J. Wahl [14].

\section{References}

[1] Artin, M., On the solution of analytic equations, Inv. Math., 5 (1968), 277-291.

[2] - Theorème de Représentabilité pour les Espaces Algébriques, Les Présse de L'Universite de Montreal, 1973.

[3] Berthelot, P., Classification topologique universelle des singularités, d'après F. Pham, Séminaire de Géométrie Analytique (astérisque 16), Société Mathématique de France, 1974.

[4] Grothendieck, A., Revétements Etale et Groupes Fondamental, Séminaire de Géométrie Algebrique, 1960-61.

[5] Hironoka, H., Resolution of singularities of an algebraic variety over a field of characteristic zero, Ann. of Math., 79 (1964), 109-326.

[6] Hironaka, $\mathrm{H}$. and Rossi, $\mathrm{H}$., On the equivalence of imbeddings of exceptional complex spaces, Math. Ann., 156 (1964), 313-333.

[7] Kawamata, Y., On deformations of compactifiable complex manifolds, to appear in Math. Ann.

[8] Kempf, G., Kundsen, F., Mumford, D. and SaintDonat, B., Toroidal Embeddings I, Lecture Note in Mathematics, 339 (1973), Springer.

[9] Pourcin, G., Théorème de Douady au-dessus de S, Ann. Scuola Norm. Sup. Pisa (3), 23 (1969), 451-459.

[10] Speder, J.-P., Equisingularite et conditions de Whitney, Amer. J. Math., 97 (1975), 571-588.

[11] Teissier, B., Cycle evanescents, sections planes et conditions de Whitney, Singularite a Cargese (asterisque 7 et 8), Societe Mathematique de France, 1973.

[12] - Introduction to equisingularity problems, Algebraic Geometry (Arcata 1974), 593-632, Proceedings of symposia in Pure Mathematics, 29, Amer. Math. Soc, 1975.

[13] Varchenko, A. N., Zeta-function of monodromy and Newtons diagram, Inv. Math., 37 (1976), 253-262.

[14] Wahl, J., Equisingular deformations of plane algebroid curves, Transactions of $A$. $M$. S., 193 (1974), 143-170.

[15] Wahl. J., Equisingular deformations of normal surface singularities I, Ann. of Math., 104 (1976), 325-356. 